\title{
A General Framework for Infrastructure System Reliability Modelling and Analysis
}

\author{
Payam Mokhtarian ${ }^{\text {ab* }}$ \\ Mohammad-Reza Namazi-Rad ${ }^{\mathrm{b}}$ \\ Tin Kin $\mathrm{Ho}^{\mathrm{b}}$
}

\begin{abstract}
An infrastructure system is inherently complex, with layers of both defined and subtle interfaces with other infrastructure systems and human users. High availability is desired, which implies stringent requirements on reliability and safety. Reliability analysis typically starts at component or sub-system level and aggregates through the system functional hierarchy. Because of the system complexity, incorporating occurrences of all possible interactions and scenarios is not always practical and failure data is often limited. Moreover, there are unobserved events among the sub-systems distributing either randomly or with temporal trend. To facilitate reliability analysis amid the complex environment and uncertain data, this paper proposes a general framework on modelling and aggregating reliability for complex systems with distinctive statistical approaches. The underlying principles will be illustrated and the suitability of the proposed techniques with respect to the data available will be discussed. This study contributes to the assurance of life-long productivity of infrastructure systems.
\end{abstract}

Key words: Infrastructure System; Reliability; Statistical Modelling.

\section{Introduction}

The term reliability is used generally to express a certain degree of assurance that a component or a system will operate successfully or at least at a desired level in a specified condition during a certain period of time ${ }^{1}$. If a component fails, this does not necessarily imply that it is unreliable or consequently it makes the system unreliable or unstable. Main concerns are how frequently failures at the component level occur in a specified time period and how much the overall reliability of a certain system is affected by the failures at the component level. Generally, reliability of a component depends on three functions: i) technical reliability of a component refers to nature of the component and this reliability is assumed to be a constant value, ii) mission reliability depends on mission type and is not a constant value, and iii) operational reliability refers to the persons or operational systems that operate with the component ${ }^{2}$. There are some other reliability functions that can affect the total reliability of a component while the abovementioned functions are the most effective ones. To express the total reliability of a component, a number of statistical methods have been proposed. These methods are mostly based on probabilistic behaviour of the component failure obtained experimentally.

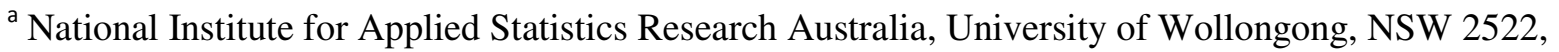
Australia

${ }^{\mathrm{b}}$ SMART Infrastructure Facility, University of Wollongong, NSW 2522, Australia.

*Corresponding author: Payam Mokhtarian

http://dx.doi.org/10.14453/isngi2013.proc.32
} 
The proposed statistical methods (derived based on the distribution of the failures) provide information on the performance of the components. It is only sensible to use this information to decide the reliability of the system. If data are available on the performance of components, it is possible to calculate or estimate the reliability for overall system. However, it is necessary to use a reliable method to calculate or estimate the reliability of the system constructed from these components. Firstly, the structure of this system must be defined in terms of its components. This is attained by the structure function with the associated concepts of paths and cuts. ${ }^{1,3,4}$ If the components operate independently and have identical probabilistic mechanisms, the reliability calculation for the system is simplified. A simple and important combination of the reliability structure function is a linear combination in which the overall system reliability is the average of the components reliability. However, it is not practical to assume such a reliability structure function and non-identical components need to be considered, as well. To deal with this structure, a number of weighted and modelbased methods have been proposed ${ }^{5}$.

Considering the definition presented for both component and system, it is easier to illustrate the system structure by a one-fold nested structure in which the system is the core and components are sub-systems. Moreover, the failure term is transferred to the performance in infrastructure reliability study. Generally, all infrastructures have this hierarchy and an infrastructure and its components are assumed to be system and sub-systems, respectively. In practice, it is important to obtain the total reliability of an infrastructure in term of performance. In real world, there are a number of infrastructures operating simultaneously or sequentially as their interdependency and sequential productivity should meet the requirement of a desired service. This definition is a natural extension of the hierarchical structure of a system. Hence, the structure of a set of infrastructure is expressed by a two-fold nested structure in which all infrastructures including their components are built on a main platform. In this paper, a general framework of reliability study for such hierarchical structure from the statistical perspective is presented.

The remainder of this paper is structured as follows. In Section 2, the structure function of reliability at the infrastructure level is studied. Section 3 describes the extension on the reliability structure function at the overall infrastructures level. Finally, Section 4 concludes the paper with a summary of proposed idea and a discussion of avenues for future researches.

\section{Infrastructure Structure Functions}

Within an infrastructure consisting of $n$ components, $x_{i}$ denotes the state of the $i^{\text {th }}$ component where $x_{i}=1$ if the component is operating and $x_{i}=0$ otherwise. The state of the infrastructure is determined by the structure function $s(\mathbf{x})$ where $\mathbf{x}=\left(x_{1}, x_{2}, \ldots, x_{n}\right)$ is the vector of component states. The vector $\mathbf{x}$ is a path when $s(\mathbf{x})=1$ and is a cut when $s(\mathbf{x})=$ 0 . The number of components in operation is

$$
T(\mathbf{x})=\sum_{i=1}^{n} x_{i} .
$$

The number of paths of size $k$ is denoted by $P_{k}$ and hence the number of cuts of size $k$ is $C_{k}^{n}-P_{k}$. An infrastructure structure based on the formation and operation of its components is categorised. A series infrastructure is an infrastructure in which all components must operate for an infrastructure to run and has productivity. The structure function is as follows 


$$
s(\mathbf{x})=\prod_{i=1}^{n} x_{i}
$$

In this case these is only one path, $x_{1}, x_{2}, \ldots, x_{n}$, which has size $n$ and it is the minimal path. Within a parallel infrastructure structure, only one component needs to operate for the infrastructure to run and has productivity. $s(\mathbf{x})$ is defined as follows

$$
s(\mathbf{x})=1-\prod_{i=1}^{n}\left(1-x_{i}\right)
$$

Here, every state equal to 1 is a path. Therefore, there are $2^{n}-1$ paths. This is a simple case of parallel infrastructure in which only one component is operating. With increasing the number of operating components in a parallel infrastructure, the total reliability will increase. The extension of a 1-parallel infrastructure is a $k$-out-of- $n$ infrastructure in which at least $k$ components must operate for the infrastructure to operate.

Within a composite infrastructure structure, components are both in series and parallel form. In this type of infrastructure structure, the operating parallel section denoted by $\mathbf{z}=$ $\left(z_{1}, z_{2}, \ldots, z_{m}\right)$. As a result,

$$
s(\mathbf{x})=1-\prod_{i=1}^{n-m}\left(1-y_{i}\right) ; \mathbf{y}=\left(y_{1}, y_{2}, \ldots, y_{n-m}\right),
$$

where $\mathbf{y}=\mathbf{x}-\mathbf{z}$ and $s(\mathbf{z})=\prod_{j=1}^{m} z_{j}$.

Figure 1 demonstrates three possible infrastructure structures discussed in term of components formation and operation where $\mathrm{A}, \mathrm{B}$ and $\mathrm{C}$ are possible infrastructure components.

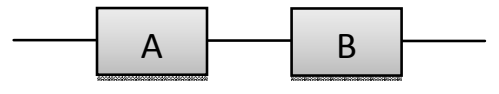

Serires Structure

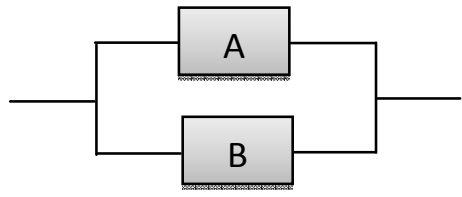

Parallel Structure

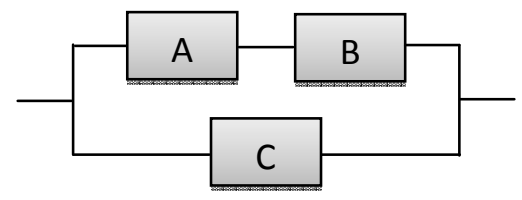

Composite Structure

Figure 1. Infrastructure structure respect to the components formation a simple infrastructure.

Reliability of each component respect to set $L$ can be defined by a probabilistic function as below,

$$
R(L)=\operatorname{Pr}(\text { Component operates in set } L) \text {, }
$$

where set $L$ is the level of satisfactory. In the context of failure analysis, this set is the lifetime or the time that component works under a certain condition. The reliability analysis of the infrastructure structures presented in Figure 1 depends on the properties of system components. The reliability function of an infrastructure is defined by its structure function considering influence of its components on the system performance. For an infrastructure consisting of $n$ components, $\mathbf{w}=\left(w_{1}, w_{2}, \ldots w_{n}\right)$ is the weight vector and $w_{i}$ is a measurement for the effects of the reliability measured for $i^{\text {th }}$ component on total reliability 
of this infrastructure, for which . Here,

denotes the whole infrastructure and the overall reliability of this infrastructure is

where is inner product of weights vector and components vector.

\section{Infrastructure Reliability Models}

This section investigates the total reliability of a set of infrastructures that operate simultaneously or sequentially. This can be expressed by a natural extension of the one-fold nested structure of an infrastructure to a two-fold nested structure. In order to study infrastructures while, $\quad$ is the number of components potentially operate within infrastructure a one-fold nested structure is considered for each of the infrastructures. When a one-fold nested structure is considered for these infrastructures on a main platform a two-fold nested structure is designed. Figure 2 illustrates the two-fold nested structure and interaction between the infrastructures as an example in which the assumed infrastructures operate in a main platform. Moreover, each infrastructure includes a number of components.

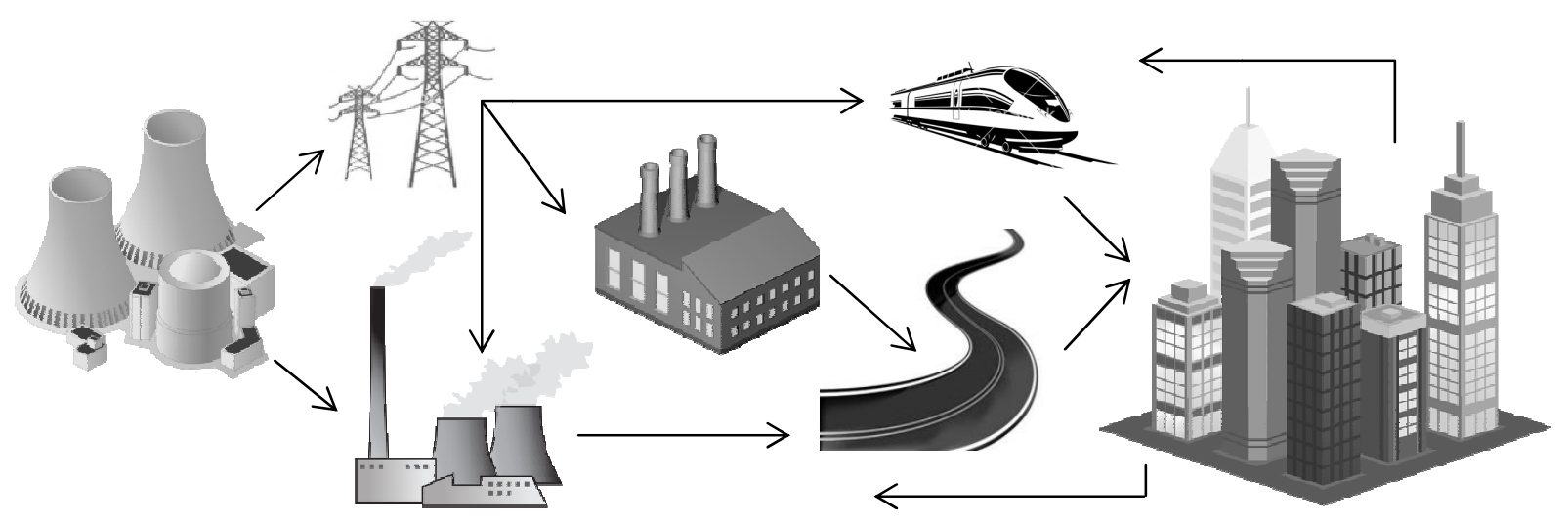

Figure 2. An example of the two-fold nested structure and interaction between the infrastructures.

Here, is the total reliability of the infrastructure while the infrastructure is the component at the main platform. In case these infrastructures are operating in series, the overall reliability is calculated using a linear weighted function as follows

where is the series effective weight of the infrastructure. In case the infrastructures are operating in parallel, the overall reliability has a cumulative linear weighted function form as below 


$$
R_{K}(\mathbf{X})=\sum_{k=1}^{K} \gamma_{k} R_{k}\left(\phi_{k}\left(\mathbf{x}_{k}\right)\right),
$$

where $\gamma_{k}$ is the parallel effective weight of the $k^{\text {th }}$ infrastructure. The overall reliability for the composite structure of infrastructures in the main platform can be calculated. In this case, the infrastructures with series operation must be considered as one infrastructure with corresponding total reliability denoted by $R_{S_{1}}(\mathbf{X}), s_{1}=1, \ldots, S_{1}$. Consequently, all sets of series infrastructures should be considered as one infrastructure. In this study, $G$ denotes the total sets of series infrastructures while the rest operate in parallel. Thus, the overall reliability of the $K$ infrastructures has a cumulative linear weighted function form as follows:

$$
R_{Q}(\mathbf{X})=\sum_{q=1}^{Q} \gamma_{q} R_{q}\left(\phi_{q}\left(\mathbf{x}_{q}\right)\right), q=1, \ldots, Q
$$

In this paper, the overall reliability of $K$ infrastructures are discussed where the formations of the infrastructures are assumed to be idealized. Some effects such as environmental, time and random effects are not considered in the basic infrastructure set up. We investigate four possible scenarios for the overall reliability of the $K$ infrastructures which are not formative only. For simplicity and without loss of generality, the scenarios under series operation between the infrastructures are studied.

\section{A. Geographically-weighted Model}

Geographic locations of the infrastructure usually affect the reliability of an infrastructure and the overall reliability of the main platform. As an instance, performance of a set of infrastructure may vary in different climates or environments. The overall reliability with geographical weight $g$ is modelled as below,

$$
R_{K}^{g}(\mathbf{X})=\sum_{k=1}^{K} g \gamma_{k} R_{k}\left(\phi_{k}\left(\mathbf{x}_{k}\right)\right)
$$

where $0 \leq g \leq 1$

\section{B. Spatial Model}

Spatial effect in this context is referred to the formation of the infrastructures in respect to each other while the serial reliability may be affected by spatial location of every consecutive infrastructure e.g., distance between infrastructures. Therefore, the overall reliability with spatial correlation is modelled as follows

$$
R_{K}^{s p}(\mathbf{X})=\sum_{k=1}^{K}\left(1-\rho_{k-1, k}^{2}\right) \gamma_{k} R_{k}\left(\phi_{k}\left(\mathbf{x}_{k}\right)\right),
$$

where $\rho_{k-1, k}$ is the spatial correlation between $(k-1)^{t h}$ and $k^{t h}$ infrastructures.

\section{Temporal Model}

Infrastructures may perform differently over time. The performance and reliability of an infrastructure can also be affected by its operating time. The element of times should be also considered in the calculation of the overall infrastructures reliability by indexing the time. The temporal model for $K$ infrastructures reliability is thus 


$$
R_{K}^{t}(\mathbf{X})=\sum_{k=1}^{K} \gamma_{k}^{t} R_{k}^{t}\left(\phi_{k}\left(\mathbf{x}_{k}\right)\right) .
$$

This model can be assumed as either stationary or non-stationary. Moreover, it can be extended to an auto-correlative model in which there time lag trend effect is also considered.

\section{Mixed-effects Model}

All the above mentioned influential effects are measurable. In practice, there is a number of effects among interactions between the infrastructures that are not measurable or observable or hard to measure in terms of accessibility and cost. These effects can be defined as random effects or mixed-effects between the infrastructures so that we can model the overall reliability of the $K$ infrastructures as below

$$
R_{K}^{M}=R_{K}(\mathbf{X})+\boldsymbol{I N F},
$$

where $R_{K}(\mathbf{X})=\sum_{k=1}^{K} \gamma_{k} R_{k}\left(\phi_{k}\left(\mathbf{x}_{k}\right)\right)$ and $\boldsymbol{I} \boldsymbol{N} \boldsymbol{F}$ is the vector of random effects defined for the $K$ infrastructures. These random effects are to be predicted using statistical modelling and estimating techniques [6]. This way, the vector of random effects is assumed to distribute symmetrically around zero.

\section{E. Hybrid Model}

Here we proposed and briefly reviewed some possible and common scenarios in infrastructure reliability analysis. These scenarios can be combined for example if the reliability of an infrastructure is to be calculated considering both spatial and temporal effects. In such a case, a Spatio-Temporal model can be used for calculating the desired reliability. These combinations are generally considered as a hybrid model. Note that random effects can be included in any type of reliability models presented in this study.

\section{Conclusion and Future Research}

In this paper we presented a general framework of the infrastructure reliability based on a two-fold nested structure. We assumed a simple model and a structure function to investigate the interaction of $K$ infrastructure in terms of performance and reliability. Moreover, a few complicated models for interactions of the infrastructures are reviewed. In future studies, complex and hybrid models should be evaluated using both numerical and graphical techniques. Employing the advanced statistical reliability methods such as nonparametric and Bayesian methods to obtain more accurate reliability estimates is the direction for future research.

\section{References}

${ }^{1}$ Zacks, S., Introduction to Reliability Analysis, Springer-Verlag, New York, 1992.

${ }^{2}$ Anasell, J.I., and Phillips, M.J., Practical Methods for reliability Data Analysis, Oxford University Press, New York, 1994. 
${ }^{3}$ Chen, S.K., Ho, T.K., and Mao, B.H., "Reliability evaluations of railway power supplies by fault-tree analysis", IET Electr. Power Appl., Vol. 1, No. 2, 2007, pp. 161-172. http://dx.doi.org/10.1049/iet-epa:20060244

${ }^{4}$ Carreras, B.A., Newman, D.E., Dobson, I. and Zeidenberg, M., "A simple model for the reliability of an infrastructure system controlled byagents", Working series paper of ASSET, University of Alaska Fairbanks, 2012.

${ }^{5}$ Wolstenholme, L.C., Reliability modelling: a statistical approach, Chapman and Hall CRC, 1999.

${ }^{6}$ McCulloch, C.E., Searle, R.S. and Neuhaus, J.M., Generalized, Linear, and Mixed Models: 2nd Edition, New York: John Wiley \& Sons, 2008. 